\title{
The integrin antagonist cilengitide increases the antitumor activity of temozolomide against malignant melanoma
}

\author{
LUCIO TENTORI $^{1}$, ANNALISA SUSANNA DORIO ${ }^{1}$, \\ ALESSIA MUZI ${ }^{1}$, PEDRO MIGUEL LACAL ${ }^{2}$, FEDERICA RUFFINI ${ }^{2}$, \\ PIERLUIGI NAVARRA ${ }^{3}$ and GRAZIA GRAZIANI ${ }^{1}$ \\ ${ }^{1}$ Department of Neuroscience, University of Rome 'Tor Vergata'; \\ ${ }^{2}$ Laboratory of Molecular Oncology, 'Istituto Dermopatico dell'Immacolata' (IDI-IRCCS); \\ ${ }^{3}$ Department of Pharmacology, Catholic University Medical School, Rome, Italy
}

Received October 18, 2007; Accepted December 3, 2007

\begin{abstract}
Inhibitors of $\alpha_{\mathrm{v}}$ integrins have been developed as anti-angiogenic agents for cancer therapy and, among them, cyclic RGD-containing pentapeptides, such as cilengitide, are the most commonly used integrin antagonists. In this study, cilengitide was tested in combination with the methylating agent temozolomide (TMZ), a well-tolerated anticancer drug with favourable pharmacokinetic properties currently used for the therapy of metastatic melanoma. To this end, the influence of cilengitide and TMZ on malignant melanoma growth and endothelial cell proliferation were investigated, using in vitro and in vivo models. The results indicated that cilengitide and TMZ exerted synergistic antiproliferative effects against melanoma and endothelial cells in vitro and induced a statistically significant reduction of in vivo melanoma growth with respect to treatment with the methylating agent only. In conclusion, this study proposes the use of cilengitide in combination with TMZ for the treatment of metastatic melanoma, thereby opening novel perspectives for the use of integrin inhibitors to enhance the efficacy of chemotherapy.
\end{abstract}

\section{Introduction}

Integrins, a family of cell adhesion molecules, mediate the interactions between cells or between cells and the extracellular matrix (ECM). They are heterodimeric cell surface glycoproteins composed of non-covalently associated transmembrane subunits, $\alpha$ and $\beta$ chains, which link the cytoskeleton to ECM components. In mammals, 18 different $\alpha$

Correspondence to: Dr Grazia Graziani, Department of Neuroscience, University of Rome 'Tor Vergata', Via Montpellier 1, 00133 Rome, Italy

E-mail: graziani@uniroma2.it

Key words: integrin, cilengitide, temozolomide, malignant melanoma and eight different $\beta$ subunits associate, giving rise to $>20$ receptors, capable of recognizing one or more ligands (1).

It is well established that integrin signaling plays an important role in tumor angiogenesis and metastasis. In particular, $\alpha_{v} \beta_{3}$ and $\alpha_{v} \beta_{5}$ integrins are highly expressed on activated endothelial cells, modulating cell migration and survival during angiogenesis, and in tumor cells, facilitating cell invasion and movement across blood vessels due to their ability to recruit and activate matrix metallo-proteases (2). In contrast to $\alpha_{v} \beta_{5}$ which solely binds to vitronectin, $\alpha_{v} \beta_{3}$ interacts with various matrix ligands. In particular, $\alpha_{v} \beta_{3}$ binds to the Arginine-Glycine-Aspartic acid (RGD)-sequence present in vitronectin, tenascin, thrombo-spondin, fibronectin and fibrinogen (3).

Inhibitors of $\alpha_{v}$ integrins have been developed as antiangiogenic agents for cancer therapy and cyclic RGDcontaining pentapeptides are the most commonly used integrin antagonists $(4,5)$. Among these, a compound which recently entered clinical trials is cilengitide (EMD121974, cycloL-Arg-Gly-L-Asp-D-Phe-N[-Methyl]L-Val), a cyclic RGD containing peptide which binds $\alpha_{v} \beta_{3}$ and $\alpha_{v} \beta_{5}$ integrins with nanomolar affinity (3). Cilengitide selectively inhibits the $\alpha_{v} \beta_{3}$ integrin binding to vitronectin with an $\mathrm{IC}_{50}$ value of $0.58-1 \mathrm{nM}$ and also antagonizes the $\alpha_{\mathrm{v}} \beta_{5}$ integrin binding to vitronectin with a high affinity $\left(\mathrm{IC}_{50} 37-140 \mathrm{nM}\right)(6,7)$. Preclinical studies indicated that cilengitide blocks tumor growth by inhibiting tumor angiogenesis. However, the compound may have antitumor effects that are independent of tumor vascularization (8-11). In phase I studies, the drug did not show any dose-limiting toxicity in solid tumors (7) and glioma $(12,13)$. Several phase II clinical trials have been performed for the treatment of a variety of cancer types including prostatic $(14,15)$, pancreatic (16), melanoma (17) and glioblastoma $(18,19)$.

Among the different solid tumors, malignant melanoma expresses $\alpha_{\mathrm{v}}$ integrins, that activate and control signaling pathways which enable tumor cells to switch from stationary to a migratory and invasive phenotype (20). The treatment of metastatic melanoma is still unsatisfactory, with a 5-year survival of treated patients ranging from 3 to $14 \%$. The 

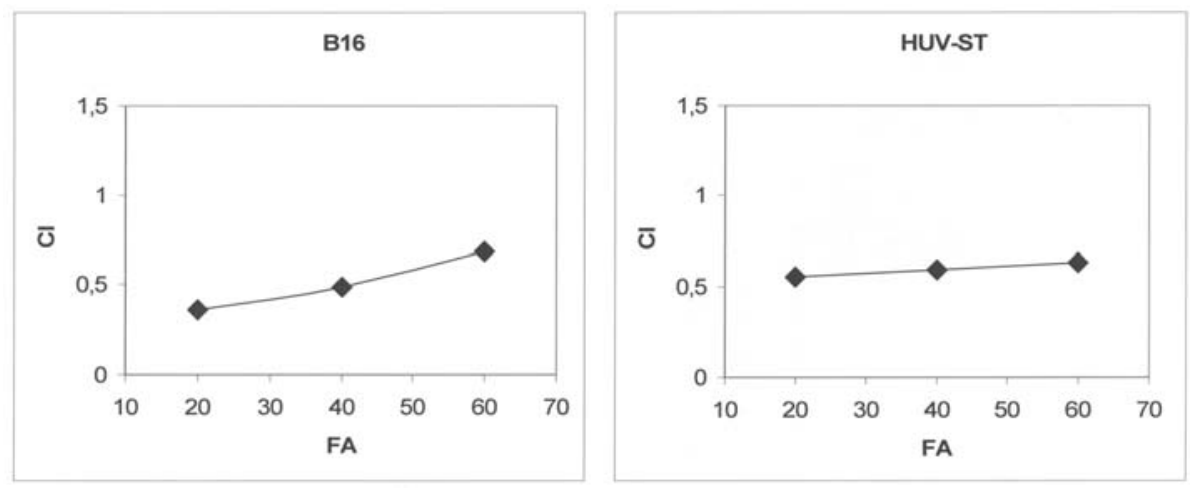

Figure 1. Synergistic effect of the TMZ and cilengitide combination in melanoma or endothelial cells. The chemosensitivity of melanoma (B16) or endothelial cells (HUV-ST) to TMZ or cilengitide as single agents, or in combination, was assessed by colony-formation assay. Cells were treated with TMZ + cilengitide at fixed molar ratios (1, 0.5, 0.2 and 0.1 times the $\mathrm{IC}_{50}$ concentration for each drug). Colony-forming ability was evaluated after $10-14$ days. A combination index (CI) fraction-affected (Fa) plots of interactions between TMZ and cilengitide were generated by computer analysis using Calcusyn software Version 2.0. $\mathrm{CI}$ indicates a synergistic $(\mathrm{CI}<1)$, additive $(\mathrm{CI}=1)$ or antagonistic effect $(\mathrm{CI}>1)$.

methylating agent dacarbazine (DTIC), is historically the reference drug for the treatment of malignant melanoma and recently its congener temozolomide (TMZ) has in part replaced DTIC due to a number of favourable properties. TMZ is a lipophilic compound with a high oral bio-availability, penetration of the blood-brain barrier and mild to moderate adverse effects (21). Although response rates were comparable, TMZ has been shown to improve progression-free survival compared to DTIC, to allow a better quality of life and to reduce the incidence of CNS relapse in patients affected by metastatic melanoma $(22,23)$. To enhance efficacy and counteract tumor resistance, TMZ is currently used in combination therapy with other chemotherapeutic agents and clinical trials, recently completed or still underway, are evaluating the efficacy of TMZ treatment in combination with inhibitors of DNA repair (24-26). In the present study, we demonstrated that cilengitide is capable of increasing the anti-melanoma activity of TMZ with no increase of systemic toxicity.

\section{Materials and methods}

Cell lines. The murine melanoma cell line B16 of C57BL/6 $\left(\mathrm{H}-2^{\mathrm{b}} / \mathrm{H}-2^{\mathrm{b}}\right.$ ) origin (ATCC, Manassas, VA) was cultured in RPMI-1640 containing 10\% fetal calf serum (Sigma-Aldrich, Milan, Italy), $2 \mathrm{mM}$ L-glutamine, $100 \mathrm{units} / \mathrm{ml}$ penicillin and $100 \mu \mathrm{g} / \mathrm{ml}$ streptomycin (Sigma-Aldrich), at $37^{\circ} \mathrm{C}$ in a $5 \%$ $\mathrm{CO}_{2}$ humidified atmosphere.

The immortalized human endothelial cell line HUV-ST was generated as previously described (27). Cells were maintained in culture in endothelial growth factor medium (EGM-2, Clonetics, BioWhittaker Inc, Walkersville, MD) and supplemented with $0.4 \mathrm{mg} / \mathrm{ml}$ geneticin and $5 \mu \mathrm{g} / \mathrm{ml}$ puromycin.

Colony-formation assay. Cells were seeded in triplicate into 6-well plates $\left(2 \times 10^{2} /\right.$ well $)$ and, after overnight incubation, were treated with TMZ (Schering-Plough, Kenilworth, NJ, USA, 15-500 $\mu \mathrm{M}$ ) or cilengitide (Merck, KGaA, Darmstadt, Germany, 0.01-10 $\mu \mathrm{M}$ ). Cells were cultured to allow colony formation and, after 10-14 days, colonies were fixed and stained with rhodamine B basic violet 10 (Sigma-Aldrich). Only colonies comprising $>50$ cells were scored as survival colonies. Chemosensitivity was evaluated in terms of $\mathrm{IC}_{50}$, i.e. the concentration of the drug capable of inhibiting the colony-forming ability by $50 \%$.

To evaluate whether the combination of $\mathrm{TMZ}+$ cilengitide was synergic, cells were exposed to TMZ or cilengitide alone or in combination at fixed equipotent ratios (corresponding to $1,0.5,0.2$ and 0.1 times the $\mathrm{IC}_{50}$ for each drug). The doseeffect curves were analyzed by the median-effect method of Chou and Talalay using the Calcusyn software (Biosoft, Cambridge, UK). The combination index (CI) indicates a quantitative measure of the degree of drug interaction in terms of synergistic $(\mathrm{CI}<1)$, additive $(\mathrm{CI}=1)$ or antagonistic effect $(\mathrm{Cl}>1)$. Graphs were generated, plotting $\mathrm{CI}$ as a function of the fraction of cells affected $(\mathrm{Fa})$ by the dose of the drugs.

Cell adhesion assays. Solid support was prepared by coating immunological 96-multiwell plates with $10 \mu \mathrm{g} / \mathrm{ml}$ vitronectin (Sigma-Aldrich) in PBS for $18 \mathrm{~h}$ and blocking with $3 \%$ BSA in PBS for $2 \mathrm{~h}$. B16 cells were plated in serum-free medium supplemented with $0.1 \% \mathrm{BSA}$ at a concentration of $4 \times 10^{4}$ cells/well. After incubation at $37^{\circ} \mathrm{C}$ for $45 \mathrm{~min}$, the nonadherent cells were washed out with PBS, whereas the attached cells were fixed with $3 \%$ formaldehyde and stained with $0.5 \%$ crystal violet. The attachment efficiency was determined by a quantitative dye extraction and the spectrophotometric measurement of the absorbance at $595 \mathrm{~nm}$.

In vivo studies on antitumor activity. Drug toxicity was evaluated by treating intact mice (5/group) with the compounds under study, used as single agents or in combination. Body weight was measured three times weekly and survival was recorded for 3 weeks after the last treatment. Toxicity was assessed on the basis of apparent drug-related deaths (within 7 days after the last treatment) and net body weight loss [i.e., (initial weight - lowest weight)/initial weight x 100\%].

B16 cells $\left(2.5 \times 10^{5}\right)$ were inoculated intra-muscle (i.m.) in seven week-old male C57BL/6 (8/group, Charles River, Calco, Italy). Tumors were measured with a caliper and the treatment was started when the nodules reached $200-250 \mathrm{~mm}^{3}$. 


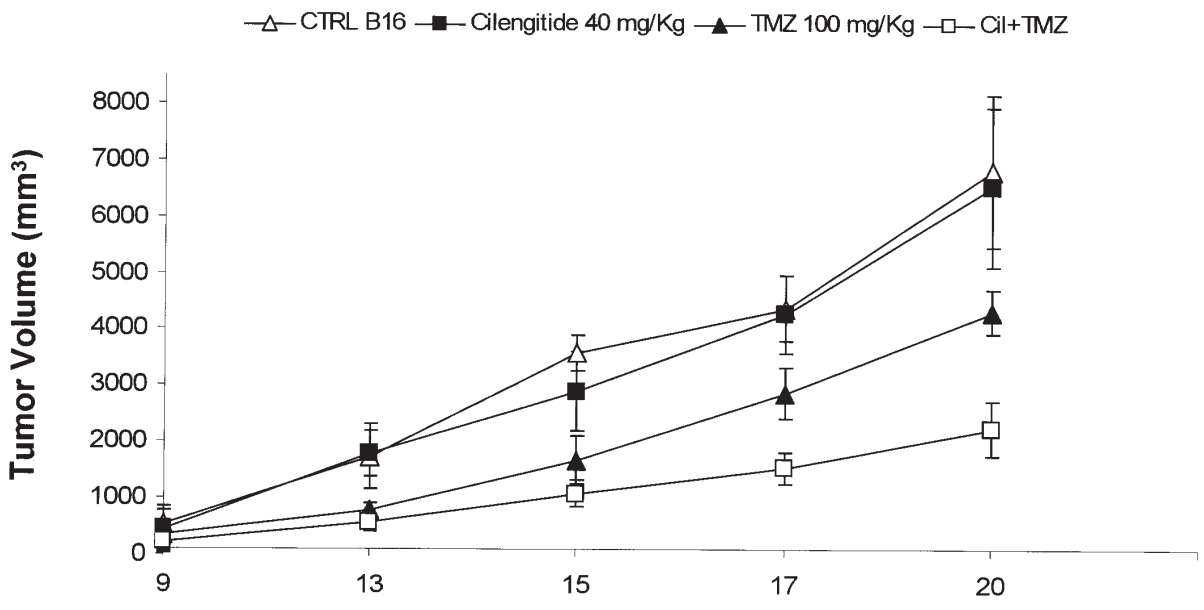

\section{Days}

Figure 2. Cilengitide increases the antitumor activity of TMZ against B16 melanoma. Treatment (TMZ $100 \mathrm{mg} / \mathrm{kg} / \mathrm{day} / \mathrm{i} . \mathrm{p}$. for 5 days or cilengitide $30 \mathrm{mg} /$ $\mathrm{kg} /$ day/i.p. for 5 days) started on day 6, when the volume of tumor nodules reached 200-250 $\mathrm{mm}^{3}$. Symbols represent the means of tumor nodule volumes determined in 8 animals for each group every 2-4 days. Bars: \pm SD. Statistical analysis of tumor growth was performed by one-way analysis of variance (ANOVA) followed by Bonferroni test for multiple comparisons. Differences between TMZ + cilengitide versus control or cilengitide groups were statistically significant starting from day 13 onward $(\mathrm{P}<0.05)$; differences between $\mathrm{TMZ}+$ cilengitide versus $\mathrm{TMZ}$ were statistically significant starting from day 17 onward $(\mathrm{P}<0.05)$; differences between $\mathrm{TMZ}$ versus control or cilengitide groups were statistically significant starting from day 13 onward $(\mathrm{P}<0.05)$.

Tumor growth was monitored by measuring tumor nodules every 2-4 days for 3 weeks.

TMZ (Shering Plough) was dissolved in dimethylsulfoxide, diluted in saline $(5 \mathrm{mg} / \mathrm{ml})$ and administered intraperitoneally (i.p.) at $100 \mathrm{mg} / \mathrm{kg} / \mathrm{dx} 5 \mathrm{~d}$. Cilengitide (Merck $\mathrm{KgaA})$ was dissolved in sterile physiological saline $(5 \mathrm{mg} / \mathrm{ml})$ and administered i.p. at $40 \mathrm{mg} / \mathrm{kg} / \mathrm{dx} 5 \mathrm{~d}$, a dose corresponding to $\sim 1 / 2$ of $\mathrm{LD}_{10}$ (7). Control mice were always treated with vehicles. The results of the in vivo tumor growth were analyzed by one-way analysis of variance (ANOVA) for multiple comparisons followed by a Bonferroni test (Primer of Biostatistics Statistical Software Program, McGraw-Hill Medical, USA). A P-value of $<0.05$ was considered significant.

All procedures involving animals and their care were performed in compliance with the national and international guidelines (European Economy Community Council Directive 86/109, OLJ318, Dec. 1, 1987).

\section{Results}

Cilengitide increases the antiproliferative effect of TMZ against melanoma and endothelial cells. B16 melanoma cells were exposed to 0.01-10 $\mu \mathrm{M}$ cilengitide and an analysis of the long-term survival of the drug-treated cells, by colony-formation assay, indicated that the drug induced a dose-dependent reduction of cell growth with an $\mathrm{IC}_{50}$ of $0.2 \pm 0.07 \mu \mathrm{M}$, while the cilengitide $\mathrm{IC}_{50}$ for $\mathrm{B} 16$ adhesion to vitronectin was $8 \pm 1.5 \mu \mathrm{M}$.

To evaluate the influence of cilengitide on endothelial cell growth, an immortalized human endothelial cell line generated in our laboratory from HUVEC was used. This cell line (HUV-ST) possesses a stabilized telomere length and an increased proliferation rate with respect to parental cells, but it is not tumorigenic and displays all major endothelial phenotypic markers (27). Notably, HUV-ST cells overexpress the tumor endothelial marker TEM-1, which is regarded as the most differentially expressed molecule in tumor-derived endothelium versus normal-derived endothelium. Therefore, the immortalized human endothelial line HUV-ST is a suitable model for studying the efficacy of anti-neovascular therapy and mimicking proliferating neovascular endothelial cells associated to the tumor mass. An analysis of cell growth showed that cilengitide reduced the colony-forming ability of endothelial cells with an $\mathrm{IC}_{50}$ of $6.7 \pm 1.2 \mu \mathrm{M}$, a concentration which approximately corresponds to the $\mathrm{IC}_{50}$ for HUVEC attachment on vitronectin (10).

The TMZ $\mathrm{IC}_{50}$ of B16 and HUV-ST cells were $115 \pm 12$ and $77 \pm 7 \mu \mathrm{M}$, respectively, according to previous studies $(27,28)$. Cells were then treated with TMZ and cilengitide in combination at fixed equipotent ratios selected on the basis of the $\mathrm{IC}_{50}$ values of each cell line, as described in Materials and methods. Fig. 1 shows the results of the median effect analysis using Calcusyn software. The CI values indicate that the combination of $\mathrm{TMZ}+$ cilengitide was synergistic in the two cell lines.

Systemic administration of cilengitide increases the antitumor activity of TMZ against melanoma. The antitumor activity of the drug combination (cilengitide $40 \mathrm{mg} / \mathrm{kg} /$ day/i.p. + TMZ $100 \mathrm{mg} / \mathrm{kg} /$ day/i.p., for 5 days), was tested in B 16 melanoma growing i.m. in C57/BL6 mice and compared to the effects induced by TMZ or cilengitide, when used as single agents. The treatment produced animal body weight loss $<10 \%$ of the original weight and all mice recovered the initial body weight one week after treatment. The results of multiple comparisons between groups using ANOVA analysis of variance show that, starting from day 17 onward, cilengitide significantly enhanced $(\mathrm{P}<0.05)$ the antitumor effect of TMZ, while the treatment with cilengitide, used as a single agent, did not affect tumor growth (Fig. 2). 


\section{Discussion}

In the present study we demonstrated for the first time that the administration of the $\alpha_{v} \beta$-integrin antagonist cilengitide enhances the antitumor activity of TMZ against malignant melanoma. In particular, the combination of cilengitide and TMZ showed synergistic antiproliferative effects against melanoma and endothelial cells in vitro and induced a statistically significant reduction of in vivo melanoma growth with respect to treatment with the methylating agent alone.

The rationale of combining cilengitide with TMZ relies on their common activity against melanoma. While the mechanism underlying the antitumor activity of cilengitide primarily relies on the inhibition of tumor angiogenesis through the disruption of the integrin-ligand interaction in endothelial cells, TMZ methylates DNA generating a wide spectrum of base adducts mainly represented by N7-methylguanine, N3-methyladenine and $\mathrm{O}^{6}$-methylguanine (29). Despite being produced in low amounts, $\mathrm{O}^{6}$-methylguanine is generally considered the main cytotoxic lesion produced by TMZ. If not repaired by $\mathrm{O}^{6}$ alkylguanine DNA alkyltransferase (AGT), $\mathrm{O}^{6}$-methylguanine inappropriately pairs with thymine and the resultant mismatches trigger the intervention of the mismatch repair system (MR), which removes the improperly paired pyrimidine to re-insert it again opposite the $\mathrm{O}^{6}$-methylguanine. Repeated cycles of MR-mediated excision/re-synthesis eventually provoke DNA nicks with apoptosis induction and growth arrest. Unfortunately, resistance to TMZ occurs quite frequently and is due to high AGT levels or to the functional defects of MR, soliciting a therapeutic approach that may counteract the emergence of drug resistance possibly without worsening the advantageous toxicological profile of the methylating agent (30). In this regard, it should be noted that the administration of cilengitide in combination with TMZ did not increase systemic toxicity.

The interest in the use of integrin inhibitors for cancer therapy has lately been revived by recent phase I or II clinical studies in which cilengitide has been safely used as a single agent or in combination with chemotherapy $(12,16)$. Protracted treatment with cilengitide induced partial or complete responses in patients affected by recurrent malignant glioma (13). The lack of the in vivo effect of cilengitide as a single agent observed in the melanoma model used for this study is likely due to the limited time frame of the drug administration (i.e. a single 5-day cycle), which has been adopted to mimic the schedule currently approved for the use of TMZ in the clinic.

The enhancing effect of the TMZ antitumor activity induced by a short course of cilengitide observed in the in vivo preclinical model is likely due to the well-recognized antiangiogenic effect of cilengitide $(31,32)$. Besides its direct effect on tumor cells, TMZ has been shown to possess antiangiogenic properties, especially when used in low doses (33). A possible modulation of the AGT activity by pretreatment with cilengitide cannot be invoked to explain the synergistic effect of the combination TMZ + cilengitide since the B16 and HUV-ST cell lines possess an almost undetectable basal activity of the repair enzyme $(27,34)$.

In conclusion, this study suggests the use of cilengitide in combination with TMZ for the treatment of metastatic melanoma, thereby opening novel perspectives for the use of integrin inhibitors not only in an adjuvant setting to prevent tumor spreading but also to enhance the efficacy of chemotherapy.

\section{Acknowledgements}

This work was supported by grants from the Italian Ministry of Education and Research ['Programmi di Ricerca scientifica di rilevante Interesse Nazionale' (PRIN) projects to GG and LT].

\section{References}

1. Hynes RO: Integrins: bidirectional, allosteric signaling machines. Cell 110: 673-687, 2002.

2. Jin $\mathrm{H}$ and Varner J: Integrins: roles in cancer development and as treatment targets. Br J Cancer 90: 561-565, 2004.

3. Xiong JP, Stehle T, Zhang R, Joachimiak A, Frech M, Goodman SL and Arnaout MA: Crystal structure of the extracellular segment of integrin alphaVbeta3 in complex with an Arg-Gly-Asp ligand. Science 296: 151-155, 2002.

4. Dechantsreiter MA, Planker E, Matha B, et al: N-methylated cyclic RGD peptides as highly active and selective alpha(v) beta(3) integrin antagonists. J Med Chem 42: 3033-3040, 1999.

5. Cai $\mathrm{W}$ and Chen X: Anti-angiogenic cancer therapy based on integrin alphavbeta3 antagonism. Anticancer Agents Med Chem 6: 407-428, 2006

6. Smith JW: Cilengitide Merck. Curr Opin Investig Drugs 4: 741-745, 2003.

7. Eskens FA, Dumez H, Hoekstra R, et al: Phase I and pharmacokinetic study of continuous twice weekly intravenous administration of Cilengitide (EMD 121974), a novel inhibitor of the integrins alphavbeta 3 and alphavbeta5 in patients with advanced solid tumours. Eur J Cancer 39: 917-926, 2003.

8. Mitjans F, Meyer T, Fittschen C, et al: In vivo therapy of malignant melanoma by means of antagonists of alphav integrins. Int J Cancer 87: 716-723, 2000.

9. Taga T, Suzuki A, Gonzalez-Gomez I, et al: alpha v-integrin antagonist EMD 121974 induces apoptosis in brain tumor cells growing on vitronectin and tenascin. Int J Cancer 98: 690-697, 2002.

10. Nisato RE, Tille JC, Jonczyk A, Goodman SL and Pepper MS: alphav beta 3 and alphav beta 5 integrin antagonists inhibit angiogenesis in vitro. Angiogenesis 6: 105-119, 2003.

11. Yamada S, Bu XY, Khankaldyyan V, Gonzales-Gomez I, McComb JG and Laug WE: Effect of the angiogenesis inhibitor Cilengitide (EMD 121974) on glioblastoma growth in nude mice. Neurosurgery 59: 1304-1312, 2006.

12. Stupp R and Ruegg C: Integrin inhibitors reaching the clinic. J Clin Oncol 25: 1637-1638, 2007.

13. Nabors LB, Mikkelsen T, Rosenfeld SS, et al: Phase I and correlative biology study of cilengitide in patients with recurrent malignant glioma. J Clin Oncol 25: 1651-1657, 2007

14. Beekman KW, Colevas AD, Cooney K, et al: Phase II evaluations of cilengitide in asymptomatic patients with androgen-independent prostate cancer: scientific rationale and study design. Clin Genitourin Cancer 4: 299-302, 2006.

15. Bradley DA, Dunn R, Ryan C, et al: EMD121974 (NSC 707544, cilengitide) in asymptomatic metastatic androgen independent prostate cancer (AIPCa) patients (pts): A randomized trial by the prostate cancer clinical trials consortium (NCI 6372). J Clin Oncol 25: 5137, 2007

16. Friess H, Langrehr JM, Oettle H, et al: A randomized multicenter phase II trial of the angiogenesis inhibitor Cilengitide (EMD 121974) and gemcitabine compared with gemcitabine alone in advanced unresectable pancreatic cancer. BMC Cancer 6: 285-296, 2006.

17. Kim KB, Diwan AH, Papadopoulos NE, et al: A randomized phase II study of EMD 121974 in patients (pts) with metastatic melanoma (MM). J Clin Oncol 25: 8548, 2007.

18. Stupp R, Goldbrunner R, Neyns B, et al: Phase I/IIa trial of cilengitide (EMD121974) and temozolomide with concomitant radiotherapy, followed by temozolomide and cilengitide maintenance therapy in patients (pts) with newly diagnosed glioblastoma (GBM). J Clin Oncol 25: 2000, 2007. 
19. Reardon D, Fink K, Nabors B, et al: Phase IIa trial of cilengitide (EMD121974) single-agent therapy in patients (pts) with recurrent glioblastoma (GBM): EMD 121974-009. J Clin Oncol 25: 2002, 2007.

20. Seftor RE, Seftor EA and Hendrix MJ: Molecular role(s) for integrins in human melanoma invasion. Cancer Metastasis Rev 18: 359-375, 1999.

21. Danson SJ and Middleton MR: Temozolomide: a novel oral alkylating agent. Expert Rev Anticancer Ther 1: 13-19, 2001.

22. Middleton MR, Grob JJ, Aaronson N, et al: Randomized phase III study of temozolomide versus dacarbazine in the treatment of patients with advanced metastatic malignant melanoma. J Clin Oncol 18: 158-166, 2000.

23. Quirbt I, Verma S, Petrella T, Bak K and Charette M: The members of the melanoma disease site group of cancer care Ontario's program in evidence-based care: Temozolomide for the treatment of metastatic melanoma. Curr Oncol 14: 27-33, 2007.

24. Tentori L and Graziani G: Chemopotentiation by PARP inhibitors in cancer therapy. Pharmacol Res 52: 25-33, 2005.

25. Plummer ER: Inhibition of poly (ADP-ribose) polymerase in cancer. Curr Opin Pharmacol 6: 364-368, 2006.

26. Kefford RF, Mortimer P, Harris PA, et al: Randomized trial of the combination of lomeguatrib and temozolomide compared with temozolomide alone in chemotherapy naive patients with metastatic cutaneous melanoma. J Clin Oncol 25: 2540-2545, 2007.

27. Tentori L, Vergati M, Muzi A, et al: Generation of an immortalized human endothelial cell line as a model of neovascular proliferating endothelial cells to assess chemosensitivity to anticancer drugs. Int J Oncol 27: 525-535, 2005.
28. Tentori L, Leonetti C, Scarsella M, et al: Systemic administration of GPI 15427, a novel poly(ADP-ribose) polymerase-1 inhibitor, increases the antitumor activity of temozolomide against intracranial melanoma, glioma, lymphoma. Clin Cancer Res 9: 5370-5379, 2003.

29. Newlands ES, Stevens MF, Wedge SR, Wheelhouse RT and Brock C: Temozolomide: a review of its discovery, chemical properties, pre-clinical development and clinical trials. Cancer Treat Rev 23: 35-61, 1997.

30. Tentori L and Graziani G: Pharmacological strategies to increase the antitumor activity of methylating agents. Curr Med Chem 9: 1285-1301, 2002.

31. Albert JM, Cao C, Geng L, Leavitt L, Hallahan DE and Lu B Integrin alpha $\mathrm{v}$ beta 3 antagonist Cilengitide enhances efficacy of radiotherapy in endothelial cell and non-small-cell lung cancer models. Int J Radiat Oncol Biol Phys 65: 1536-1543, 2006.

32. Loges S, Butzal M, Otten J, et al: Cilengitide inhibits proliferation and differentiation of human endothelial progenitor cells in vitro. Biochem Biophys Res Commun 357: 1016-1020, 2007.

33. Kurzen H, Schmitt S, Naher H and Mohler T: Inhibition of angiogenesis by non-toxic doses of temozolomide. Anticancer Drugs 14: 515-522, 2003.

34. Godeneche D, Rapp M, Thierry A, Laval F, Madelmont JC, Chollet P and Veyre A: DNA damage induced by a new 2chloroethyl nitrosourea on malignant melanoma cells. Cancer Res 50: 5898-5903, 1990. 